\title{
Two-Year Follow-Up after Contact Force Sensing Radiofrequency Catheter and Second-Generation Cryoballoon Ablation for Paroxysmal Atrial Fibrillation: A Comparative Single Centre Study
}

\author{
Attila Kardos, ${ }^{1}$ Zsuzsanna Kis, ${ }^{2}$ Zoltan Som, ${ }^{1}$ Zsofia Nagy, ${ }^{1}$ and Csaba Foldesi ${ }^{1}$ \\ ${ }^{1}$ Department of Cardiac Electrophysiology, Gottsegen Gyorgy National Institute of Cardiology, Haller Street 29, \\ Budapest 1096, Hungary \\ ${ }^{2}$ Department of Clinical Electrophysiology, Erasmus Medical Center, 's-Gravendijkwal 230, Postbus 2040, \\ 3015 CE Rotterdam, Netherlands
}

Correspondence should be addressed to Attila Kardos; drkardosattila@gmail.com

Received 25 January 2016; Revised 19 April 2016; Accepted 3 May 2016

Academic Editor: Christof Kolb

Copyright (c) 2016 Attila Kardos et al. This is an open access article distributed under the Creative Commons Attribution License, which permits unrestricted use, distribution, and reproduction in any medium, provided the original work is properly cited.

\begin{abstract}
Background. There are little comparative data on catheter ablation of paroxysmal atrial fibrillation (AF) using the contact force radiofrequency (CF-RF) catheter versus the second-generation cryoballoon (CB2). Methods and results. This is a single center, retrospective, nonrandomized study of 98 patients with symptomatic, drug-refractory paroxysmal AF who underwent their first PVI ablation using either the CB2 $(n=40)$ or CF-RF $(n=58)$. The mean age was 60 years with $63 \%$ men, a mean LA size of $42 \mathrm{~mm}$. The procedure duration $(74 \pm 17$ versus $120 \pm 49$ minutes $p<0.05)$ was shorter for CB2 group; the fluoroscopy time (14 \pm 17 versus $16 \pm 5$ minutes, $p=0.45$ ) was similar. Complete PVI was achieved in $96 \%$ of patients with RF-CF and $98 \%$ with CB2. Phrenic nerve palsies (2 transient and 1 persistent) occurred exclusively in the CB2 group and 1 severe, nonlethal complication (pericardial tamponade) occurred in the CF-RF group. At 24-month follow-up, the success rate, defined as freedom from AF/atrial tachycardia (AT) after a single procedure without antiarrhythmic drug, was comparable in CF-RF group and CB2 group (65.5\% versus $67 \%$, resp., $\log$ rank $p=0.54)$. Conclusion. Both the $\mathrm{CB} 2$ and the RF-CF ablation appeared safe; the success rate at 2 years was comparable between both technologies.
\end{abstract}

\section{Introduction}

In transcatheter ablation of pulmonary veins (PVs), pulmonary vein isolation (PVI) has been established as an effective therapeutic option for patients with symptomatic drugrefractory paroxysmal atrial fibrillation (AF). PVI can be successfully achieved with different energy sources; radiofrequency ablation (RF) and cryoballoon (CB) ablation are the most frequently used technologies [1-6]. RF ablation by means of a focal-tip catheter in combination with a $3 \mathrm{D}$ mapping system is still the standard technique performed worldwide. With continuous improvements in catheter technology, such as contact force radiofrequency (CF-RF), additional clinical benefit during PVI is reported when compared to non-CF technology in recent prospective, nonrandomized trials. Marijon et al. reported a potential benefit of real-time contact force sensing technology (compared to conventional irrigated radiofrequency ablation catheter), in reducing $\mathrm{AF}$ recurrence during the first year after PVI in 60 patients, Jarman et al. reported the results of a total of 600 ablation procedures (200 using CF-RF), the mean follow-up was 12 months, and the use of force sensing catheters independently predicted clinical success in paroxysmal AF patients $[7,8]$. Operator experience is a determinant of outcome after PVI even in high-volume centres [9]. In recent years, the use of novel balloon based technologies such as cryoballoon ablation has emerged as an alternative approach to pointby-point technology $[5,6]$. CB technology might offer more 
reproducible and standardizable procedures by simplifying the ablation itself. There are certain practical advantages, such as reduced pain during ablation, improved catheter stability due to catheter-tissue adhesion, and ability to rapidly create circumferential lesions. Although it is clear that the patients benefit more from an experienced cryoballoon operator, Mikhaylov et al. showed that, in low-volume centres, where operators have experience in simple ablation procedures, $\mathrm{CB}$ is a safe procedure with success rates comparable to those obtained in higher-volume AF centres. This underlines that $\mathrm{CB}$ has a fast and reproducible learning curve in both highand low-volume AF ablation centres [10]. The first-generation $\mathrm{CB}$ was compared to standard RF ablation by Mugnai et al., who reported similar AF recurrence and AF episode burden after pulmonary vein isolation using conventional irrigated radiofrequency energy versus the first-generation cryoablation. Freedom from AF was reached in $63.5 \%$ of patients treated with cryothermia and $57.3 \%$ of patients treated with RF at 23-month follow-up [11]. The prospective FreezeAF trial demonstrated noninferiority of $\mathrm{CB}$ ablation versus $\mathrm{RF}$ ablation for treating patients with paroxysmal AF. The primary endpoint (freedom from atrial arrhythmia) at 12 months was achieved by $70.7 \%$ with RF and $73.6 \%$ with $\mathrm{CB}$ (multiple procedure success), including 31 redo procedures in each group (19.5\% of RF versus $19.9 \%$ of CB; $p=0.933$ ) [12].

Recently, the second-generation CB (CB2) has been introduced featuring homogeneous cooling of the entire frontal surface with increased refrigerant flow [13]. Significantly improved clinical outcome was demonstrated by Fürnkranz et al. (83.6\% versus $63.9 \%$ of arrhythmia-free survival) upon using the second-generation $\mathrm{CB}$ after a single procedure without AAD therapy after 1 year when compared to the first-generation $\mathrm{CB}$ [14]. A recently published multicentre, retrospective study of more than 1100 patients suggests that freedom from atrial fibrillation following PVI using the second-generation $\mathrm{CB}$ is superior to open irrigated, nonforce sensing RF based ablation at 12 months following a single procedure [15].

To the best of our knowledge, published data on comparison of medium- and long-term results between new generation $\mathrm{CB}$ and conventional manual CF-RF ablation are limited. The aim of this study was to compare the efficacy and safety of the new cryoballoon ablation (CB2) with the CF-RF approach in the treatment of paroxysmal AF.

\section{Methods}

This was a nonrandomized, retrospective, single centre study. From September 2012 to December 2013, 98 patients with drug resistant paroxysmal atrial fibrillation (defined as selfterminated, documented episodes lasting $<7$ days) who underwent initial PVI using either radiofrequency energy with contact force sensing ablation catheter (ThermoCool SmartTouch, Biosense Webster Inc., Diamond Bar, CA, USA; $n=58$ ) or the second-generation cryoballoon (Arctic Front Advance, Medtronic, Minneapolis, MN, USA; $n=40$ ) were enrolled. Written informed consent was obtained from all the patients. Before the procedure, all patients underwent a 2-dimensional transthoracic echocardiography to assess left ventricular ejection fraction and atrial dimensions and to rule out any structural and/or valvular disease. A transesophageal echocardiography was performed the day before ablation to analyze left atrial (LA) and PV anatomy and to rule out intracardiac thrombus formation. Antiarrhythmic medications were not stopped before the procedure.

All patients received anticoagulation therapy during the 4 weeks before and for at least 3 months after the index procedure. Periprocedurally, administration of the anticoagulation therapy was uninterrupted, regardless of the selected drug.

$\mathrm{RF}$ ablation instances were performed as follows.

Two nonsteerable long sheaths (St. Jude Medical, St Paul, MN, USA) were introduced into LA using a double transseptal puncture technique. An initial intravenous bolus of heparin $(100 \mathrm{IU} / \mathrm{kg})$ was followed by extra boluses to maintain an activated clotting time $(\mathrm{ACT})>300 \mathrm{~s}$. An electroanatomic map of the LA was performed using CARTO system (Biosense Webster Inc., Diamond Bar, CA, USA) with a circumferential mapping catheter (LassoNav ${ }^{\mathrm{TM}}$, Biosense Webster Inc., Diamond Bar, CA, USA). Antral PVI was performed with open irrigated tip $3.5 \mathrm{~mm}$ force sensing ablation catheter (Navistar Thermocool SmartTouch, Biosense Webster Inc., Diamond Bar, CA, USA) in a power-controlled mode with a power limit of $35 \mathrm{~W}$ and at a maximum temperature of $48^{\circ} \mathrm{C}$. Power was reduced to $25 \mathrm{~W}$ during ablation of LA posterior wall to prevent esophageal injury. The PVs were isolated by a circumferential lesion set. The endpoint was elimination of local bipolar electrogram during ablation with $20-40$ s lesions. The VisiTag ${ }^{\mathrm{TM}}$ module of CARTO 3 had been used in patients since January 2013; the settings during LA ablation was a modification of the settings reported by Lin et al. [16]:

(i) A minimum time of 20 seconds.

(ii) A maximum range of $4 \mathrm{~mm}$.

(iii) A force over time of $50 \%$.

(iv) A minimum force of $6 \mathrm{~g}$.

The desired minimum CF was $10 \mathrm{~g}$ for lesion formation; upper limit defined was $50 \mathrm{~g}$ force to avoid perforation.

$\mathrm{CB}$ ablation instances were performed as follows.

A single transseptal puncture was achieved under fluoroscopic guidance. After gaining the LA access, a $100 \mathrm{UI} / \mathrm{kg}$ heparin intravenous bolus was given, as well as extra boluses to maintain ACT $>300$ s. A $0.32 \mathrm{Fr}$ exchange wire $\left(\right.$ Emerald $^{\mathrm{TM}}$ Cordis, Johnson and Johnson, Diamond Bar, CA, USA) was advanced in the left superior PV and a steerable 15 Fr overthe-wire sheath (FlexCath Advance ${ }^{\mathrm{TM}}$, Medtronic, Minneapolis, MN, USA) was positioned in the left atrium. A circular mapping catheter (Achieve ${ }^{\mathrm{TM}}$, Medtronic, Minneapolis, MN, USA) together with a second-generation $28 \mathrm{~mm}$ doublewalled CB (Arctic Front Advance, Medtronic, Minneapolis, MN, USA) was then advanced and positioned in the PV ostium of each vein. Vessel occlusion was evaluated according to a semiquantitative grading ranging from grade 0 (very poor occlusion) to grade 4 (perfect occlusion) after dye injection; optimal vessel occlusion was deemed as total contrast retention with no backflow in the atrium. For each vein, cryoablation consisted of $\geq 1$ applications lasting $4 \mathrm{~min}$. 
Phrenic nerve function was monitored during right superior vein cryoablation by fluoroscopic assessment of diaphragm movement during spontaneous breathing.

All procedures were performed under deep sedation utilizing fractionated intravenous bolus of midazolam and fentanyl or continuous infusion of propofol with preservation of spontaneous breathing and continuous monitoring of oxygen saturation.

PV isolation was guided by circular mapping catheter (LassoNav, Biosense Webster Inc., Diamond Bar, CA, USA, and Achieve, Medtronic, Minneapolis, MN, USA) in both groups. Once entrance block was confirmed, pacing from all the bipoles on the circular mapping catheter was used to assess exit block by using a pacing stimulus of $10 \mathrm{~mA}$ at $2 \mathrm{~ms}$. Evidence for PV capture without conduction to LA was necessary to prove exit block. The procedures were stopped immediately after initial isolation; adenosine was not administered.

Postprocedural anticoagulation was started after sheath removal with low molecular weight heparin, or NOAC. Anticoagulation after the procedure was continued for at least three months or longer depending on the individual $\mathrm{CHA}_{2} \mathrm{DS}_{2}$-VASc score. Antiarrhythmic drugs were continued for 3 months after ablation and then were stopped.

2.1. Patient-Follow-Up. Clinical follow-up consisted of physical examinations, 12-lead ECG, and 24-hour Holter recording performed at 3, 6, and 12 months after ablation and every 6 months after the first year. At 9, 15, and 21 months, patients were asked about their AF symptoms with standardized questionnaires by telephone. We directed patients to check their pulse rate and rhythm at least once a day and to visit the outpatient clinic if they experienced relapse of AF.

A blanking period of 3 months was considered for the study. All documented episodes of atrial tachyarrhythmias lasting 30 seconds and any symptoms suggesting AF were considered as recurrence. Success rate was defined as the percentage of patients who did not have any documented AF episode or any symptoms suggesting AF recurrence after the blanking period within the follow-up period.

2.2. Statistical Analysis. Continuous measures are expressed as the mean value \pm standard deviation and were analyzed with the two-sided $t$-test after testing for normal distribution with the method of Kolmogorov and Smirnov. $\chi^{2}$ test was used for between-groups comparison. Kaplan-Meier eventfree survival analysis was conducted to assess the cumulative freedom from recurrence. A $p$ value $<0.05$ was considered statistically significant.

\section{Results}

Starting in September 2012, a total of 98 patients were included in the study.

Baseline characteristics are shown in Table 1.

Procedure time (from venous puncture to removal of sheaths) was significantly shorter in the CB2 group (74 \pm 17 minutes versus $120 \pm 49, p<0.05$ ); fluoroscopy time was similar in both groups (14 \pm 17 versus $16 \pm 5$ minutes, $p=0.45$ ). Complete PVI was achieved in 98\% (39 of 40) of cryoballoon-treated patients and in 96\% (56 of 58) of RFtreated patients. The $28 \mathrm{~mm}$ cryoballoon was used during all procedures without touch-ups. Three left common PVs (LCPVs) were identified in the 40 patients. The mean number of CB applications per PV was $1.5 \pm 0.8$ for the LSPV, $1.3 \pm 0.6$ for the LIPV, $1.5 \pm 0.8$ for the RSPV, $1.7 \pm 0.9$ for the RIPV, and $2.0 \pm 1.3$ for the LCPV, respectively.

The minimum balloon temperatures (as marker of balloon-tissue contact) measured were lower in the inferior PVs: LSPV: $-49.5 \pm 6^{\circ} \mathrm{C}$ versus LIPV: $-44.6 \pm 7^{\circ} \mathrm{C}(p<0.05)$ and RSPV: $-50 \pm 7^{\circ} \mathrm{C}$ versus RIPV: $-41 \pm 10^{\circ} \mathrm{C}(p<0.001)$, respectively.

Real-time catheter-tissue contact force and force time integral (FTI) were continuously monitored in 51 out of 58 CF-RF patients.

Significantly lower FTI values were detected on the anterior and inferior aspects of the left pulmonary veins and the posteroinferior region of the right inferior pulmonary vein as compared to the other PV regions using nonsteerable sheaths (Figure 1).

All patients completed the 12-month and the extended 24month follow-up. At 1 year, 77.5\% (45/58) in the CF-RF group and $80 \%(32 / 40)$ in the CB2 group were free from recurrent $\mathrm{AF} / \mathrm{AT}$, while at 24 months $65.6 \%(38 / 58)$ in the CF-RF group and $67.5 \%(27 / 40)$ in the CB2 group off AAD remained in sinus rhythm without statistical significance (Figure 2).

After index PVI, the previously ineffective AAD was started in all patients experiencing recurrence. A total of 22 patients unresponsive to AAD ( 15 pts from CF-RF and 7 pts from $\mathrm{CB}$ group) suffering from $\mathrm{AF}$ recurrence underwent a repeat ablation $13.3 \pm 7.8$ months after initial PVI. All repeat procedures were performed by the means of RF energy. In the CF-RF group, among 60 PVs, 37 (61\%) showed conduction gaps in 15 patients (2.5 per patient), whereas in the CB2 group, among 28 veins, 10 (35\%) showed a PV reconnection in 6 patients (1.4 per patient) $(p=0.01)$. In one CB2 patient, all the PVs were isolated despite of documented symptomatic paroxysmal atrial fibrillation episodes; non-PV foci were ablated.

Reconnection rates per vein in the CF-RF group were as follows: LSPV: 53\% (8/15), LIPV: 66\% (10/15), RSPV: 40\% (6/15), and RIPV: 87\% (13/15).

The inferior pulmonary veins (the left and right inferior PVs) were frequently reconnected in the CB2 group (86\% $12 / 14$ veins), while conduction gaps could be documented in the right and left superior PV only in one patient.

Procedural complication rate was low. One pericardial tamponade treated by pericardiocentesis in the CF-RF group was detected, with 3 phrenic nerve palsies in the CB2 group, two resolved completely before hospital discharge, and the third also did resolve after 12 months.

\section{Discussion}

As far as we know, these are the first published data with the longest follow-up to compare the feasibility and efficacy of the second-generation cryoballoon ablation in circumferential 
TABle 1: Patients' baseline characteristics (LA: left atrial, NOACs: novel oral anticoagulants).

\begin{tabular}{lccc}
\hline Patients $(n)$ & RG group (58) & CB group (40) & \\
\hline Age (years) & $61 \pm 9$ & $59 \pm 10$ & nalue \\
Female gender $n(\%)$ & $20(34)$ & $13(32.5)$ & \\
LA size (mm) & $42.1 \pm 4.6$ & $41.3 \pm 4$ & $\mathrm{~ns}$ \\
Hypertension (\%) & $30(51 \%)$ & $17(42.5)$ & $\mathrm{ns}$ \\
Type II diabetes (\%) & $3(5.1)$ & $2(5)$ & $\mathrm{ns}$ \\
Coronary artery disease (\%) & $7(12)$ & $5(12.5)$ & $\mathrm{ns}$ \\
Medication prior to ablation & & & $\mathrm{ns}$ \\
Beta blockers (\%) & $55(95)$ & $40(100)$ & $\mathrm{ns}$ \\
Propafenone (\%) & $24(41)$ & $12(30)$ & $\mathrm{ns}$ \\
Sotalol (\%) & $2(3)$ & $0(0)$ & $\mathrm{ns}$ \\
Amiodarone (\%) & $2(3)$ & $1(2.5)$ & $\mathrm{ns}$ \\
Acenocoumarol (\%) & $40(69)$ & $29(72.5)$ & \\
NOACs $(\%)$ & $5(8.6)$ & $4(10)$ & $\mathrm{ns}$ \\
\hline
\end{tabular}

Right PVs

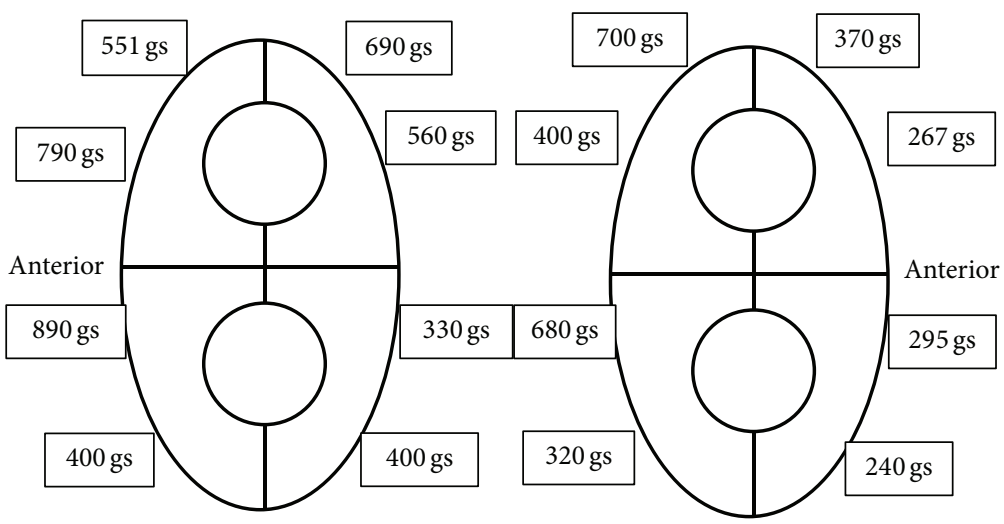

FIGURE 1: Distribution of mean force time integral (FTI) values (gs) per PV-quadrants in the CF-RF group.

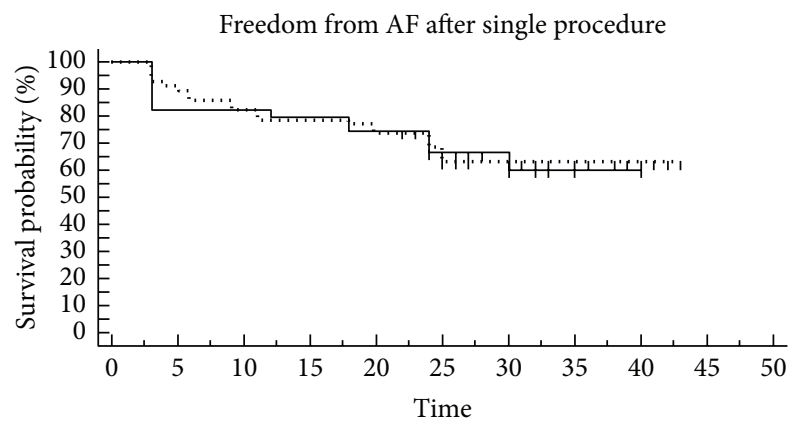

Number of those at risk

$\begin{array}{lllllllllll}\text { Group: CB } & 40 & 33 & 33 & 32 & 30 & 16 & 8 & 1 & 0 & 0 \\ \text { Group: RF } & 58 & 52 & 48 & 46 & 43 & 31 & 23 & 11 & 4 & 0\end{array}$

Ablation group

$-\mathrm{CB}$

FIGURE 2: Kaplan-Meier estimation of the time to AF recurrence after single ablation in the CF-RF and CB ablation groups (CB group: solid line; RF group: dotted line). 
PV isolation with contact force sensing RF ablation in paroxysmal atrial fibrillation after 2 years. Though the results of this single centre, retrospective study should be interpreted carefully, several findings are noteworthy.

First, in our study no differences of medium- and longterm success rates were found between both CF-RF and CB2 groups (76.5\% versus $81 \%$ at 12 months and $65.5 \%$ and $67.5 \%$ at 24 months). Our results are similar to those of the SMART-AF trial, where the 12-month success rate was $74 \%$ with CF-RF catheters [17] while several studies reported $80 \%$ or even higher clinical success with the new generation cryoballoon at 1 year $[14,18]$. In the recently published FIRE and ICE multicentre randomized study where the mean duration of follow-up was 1.5 years, the reported recurrence rates were $34.6 \%$ and $35.9 \%$ in the cryoballoon and in the radiofrequency group. Although the representation of the most advanced generation catheters was not equal in this study (first-generation CB (24\%) and CB2 (76\%) in the CB group and non-CF-RF (76\%) and CF-RF (24\%) in the RF group), no significant difference was found between the catheters used in terms of efficacy and safety [19].

The use of nonsteerable long sheath might have influenced the success rate in the CF-RF group in our study. The use of steerable sheath theoretically would improve the outcome of CF-RF ablation; these devices were designed to improve access to and contact with ablation target sites. One prospective, randomized, controlled study demonstrates that AF catheter ablation using a steerable transseptal sheath is associated with a significantly better rhythm control compared with an ablation with a nonsteerable sheath. Single procedure outcome has improved by $20 \%$ [20].

The improvements in cryoballoon cooling homogeneity found in Arctic Front Advance may address some issues that impeded long-term success of cryoballoon ablation as reported by several authors [13-15]. In fact, the CB2 might achieve significantly lower temperatures and faster isolation times in comparison with the first-generation device [14]. We aimed for single 4-min-duration freeze-thaw cycles at all PVs without bonus freeze. This concept has been applied by other operators using the CB2 [21], and even a recent report on acute procedural outcomes and short-term follow-up of CB2 ablation with 3-min duration freeze-thaw cycles showed excellent results [22].

In our study, CB2 ablation was associated with significantly shorter procedure (from venous puncture to removal of sheaths) time $(74 \pm 17$ minutes versus $120 \pm 49, p<$ 0.05), as compared to CF-RF, which is in line with most of the published comparative studies (RF versus $\mathrm{CB}$ ) in the literature $[11,19]$.

Despite AF ablation using cryoenergy and RF ablation with real-time contact force monitoring evolved dramatic improvements, arrhythmic recurrences after the index procedure remain relatively frequent and in most cases are related to $\mathrm{PV}$ reconnections $[23,24]$.

Ciconte et al. reported a significantly higher rate of late $\mathrm{PV}$ reconnection following CF-RF ablation when compared with CB2 ablation 9.8-11.7 months after the index procedure. The reported rate of PV reconnection (20.4\% versus 36.1\%; 1.2 versus 1.8 per patient) and anatomical reconnection pattern around the pulmonary veins were different in the two groups [24]. We found higher reconnection rates both in the CF-RF group (61\%, 2.5 per patient) and in the CB2 group (35\%, 1.4 per patient) $(p=0.01)$.

In the CB2 group, dominantly the inferior veins reconnected, superior PV reconnection was detected only in 1 out of 7 patients. The explanation is probably the straight orientation of the cryoballoon catheter towards the superior vein antrum, which allows better vessel occlusion, lower nadir temperature, and better tissue-balloon contact, while, in case of difficult occlusion of the inferior PVs, a pull-down technique is needed in order to obtain electrical isolation. Cryoballoon temperatures give reliable information about balloon-tissue contact. Minimum temperature achieved during inferior vein cryoablation was significantly decreased in our study as compared with those measured in the superior PVs.

Although acute isolation can be successfully achieved with CF-RF, late PV reconduction is found in at least one vein during redo procedures [24]. EFFICAS I study has recently showed that $65 \%$ of patients following CF-RF procedure experienced a reconnection in at least 1 PV 3 months after ablation, which was related to lower CF values. We found 2.5 nonisolated PVs per patient; the predominant site of PV reconnection was the anterior-inferior segments of the left PVs, as well as the inferior and inferoposterior part of the RIPV. The use of nonsteerable sheath and the longer followup (13.3 \pm 7.8 months) may be responsible for the different reconnection rates observed in the present study. The lowest FTI values on all left anterior positions are in line with the findings reported in EFFICAS I study, where a deflectable sheath was used in $46 \%$ of the patients. The extended use of steerable sheath may result in higher CF values and lower late conduction gaps upon targeting these PV segments.

4.1. Periprocedural Complications. No death and no stroke or TIA occurred in our study while pericardial tamponade in one patient $(1.7 \%)$ in the CF-RF group and phrenic nerve palsy in 3 patients in the CB2 group (7.5\%) were observed. The tamponade was successfully treated by pericardial drainage. Luckily, 2 phrenic nerve palsies recovered spontaneously before hospital discharge, and one recovered after 1 year follow-up; our results were similar to previously published data [19].

\section{Conclusion}

Both the second-generation cryoballoon and the contact force RF ablation appeared safe. PVI for paroxysmal AF is faster with $\mathrm{CB}$ and results in a similar single procedure success rate at 2-year follow-up as the conventional point-bypoint CF-RF.

\section{Competing Interests}

The authors declare that they have no competing interests. 


\section{References}

[1] M. Haïssaguerre, P. Jaïs, D. C. Shah et al., "Spontaneous initiation of atrial fibrillation by ectopic beats originating in the pulmonary veins," The New England Journal of Medicine, vol. 339, no. 10, pp. 659-666, 1998.

[2] C. Pappone, S. Rosanio, G. Oreto et al., "Circumferential radiofrequency ablation of pulmonary vein ostia: a new anatomic approach for curing atrial fibrillation," Circulation, vol. 102, no. 21, pp. 2619-2628, 2000.

[3] G. Lee, P. Sanders, and J. M. Kalman, "Catheter ablation of atrial arrhythmias: state of the art," The Lancet, vol. 380, no. 9852, pp. 1509-1519, 2012.

[4] K.-H. Kuck and A. Fürnkranz, "Cryoballoon ablation of atrial fibrillation," Journal of Cardiovascular Electrophysiology, vol. 21, no. 12, pp. 1427-1431, 2010.

[5] D. M. Fitzgerald, "Catheter ablation of atrial fibrillation: to freeze, or not to freeze, that is the question," Journal of Cardiovascular Electrophysiology, vol. 25, no. 1, pp. 8-10, 2014.

[6] J. G. Andrade, P. Khairy, P. G. Guerra et al., "Efficacy and safety of cryoballoon ablation for atrial fibrillation: a systematic review of published studies," Heart Rhythm, vol. 8, no. 9, pp. 1444-1451, 2011.

[7] E. Marijon, S. Fazaa, K. Narayanan et al., "Real-time contact force sensing for pulmonary vein isolation in the setting of paroxysmal atrial fibrillation: procedural and 1-year results," Journal of Cardiovascular Electrophysiology, vol. 25, no. 2, pp. 130-137, 2014.

[8] J. W. E. Jarman, S. Panikker, M. Das et al., "Relationship between contact force sensing technology and medium-term outcome of atrial fibrillation ablation: a multicenter study of 600 patients," Journal of Cardiovascular Electrophysiology, vol. 26, no. 4, pp. 378-384, 2015.

[9] A. Sairaku, Y. Yoshida, Y. Nakano et al., "Who is the operator, that is the question: a multicentre study of catheter ablation of atrial fibrillation," Europace, 2016.

[10] E. N. Mikhaylov, D. S. Lebedev, E. A. Pokushalov et al., "Outcomes of cryoballoon ablation in high- and low-volume atrial fibrillation ablation centres: a Russian Pilot Survey," BioMed Research International, vol. 2015, Article ID 591603, 8 pages, 2015.

[11] G. Mugnai, G.-B. Chierchia, C. de Asmundis et al., "Comparison of pulmonary vein isolation using cryoballoon versus conventional radiofrequency for paroxysmal atrial fibrillation," The American Journal of Cardiology, vol. 113, no. 9, pp. 1509-1513, 2014.

[12] A. Luik, A. Radzewitz, M. Kieser et al., "Cryoballoon versus open irrigated radiofrequency ablation in patients with paroxysmal atrial fibrillation," Circulation, vol. 132, no. 14, pp. 13111319, 2015.

[13] N. Coulombe, J. Paulin, and W. Su, "Improved in vivo performance of second-generation cryoballoon for pulmonary vein isolation," Journal of Cardiovascular Electrophysiology, vol. 24, no. 8, pp. 919-925, 2013.

[14] A. Fürnkranz, S. Bordignon, D. Dugo et al., "Improved 1-year clinical success rate of pulmonary vein isolation with the second-generation cryoballoon in patients with paroxysmal atrial fibrillation," Journal of Cardiovascular Electrophysiology, vol. 25, no. 8, pp. 840-844, 2014.

[15] A. Aryana, S. M. Singh, M. Kowalski et al., "Acute and longterm outcomes of catheter ablation of atrial fibrillation using the second-generation cryoballoon versus open-irrigated radiofrequency: a multicenter experience," Journal of Cardiovascular Electrophysiology, vol. 26, no. 8, pp. 832-839, 2015.

[16] T. Lin, F. Ouyang, K. H. Kuck et al., "ThermoCool SmartTouch Catheter-the evidence so far for contact force technology and the role of VisiTag module," Arrhythmia and Electropgysiology Review, vol. 3, no. 1, pp. 3-6, 2014.

[17] A. Natale, V. Y. Reddy, G. Monir et al., "Paroxysmal AF catheter ablation with a contact force sensing catheter: results of the prospective, multicenter SMART-AF trial," Journal of the American College of Cardiology, vol. 64, no. 7, pp. 647-656, 2014.

[18] G. Irfan, C. de Asmundis, G. Mugnai et al., "One-year follow-up after second-generation cryoballoon ablation for atrial fibrillation in a large cohort of patients: a single-centre experience," Europace, 2015.

[19] K.-H. Kuck, J. Brugada, A. Fürnkranz et al., "Cryoballoon or radiofrequency ablation for paroxysmal atrial fibrillation," The New England Journal of Medicine, 2016.

[20] C. Piorkowski, C. Eitel, S. Rolf et al., "Steerable versus nonsteerable sheath technology in atrial fibrillation ablation: a prospective, randomized study," Circulation: Arrhythmia and Electrophysiology, vol. 4, no. 2, pp. 157-165, 2011.

[21] E. Wissner, C.-H. Heeger, H. Grahn et al., "One-year clinical success of a "no-bonus" freeze protocol using the secondgeneration $28 \mathrm{~mm}$ cryoballoon for pulmonary vein isolation," Europace, vol. 17, no. 8, pp. 1236-1240, 2015.

[22] G. Ciconte, C. de Asmundis, J. Sieira et al., "Single 3-minute freeze for second-generation cryoballoon ablation: one-year follow-up after pulmonary vein isolation," Heart Rhythm, vol. 12, no. 4, pp. 673-680, 2015.

[23] P. Neuzil, V. Y. Reddy, J. Kautzner et al., "Electrical reconnection after pulmonary vein isolation is contingent on contact force during initial treatment: results from the EFFICAS i study," Circulation: Arrhythmia and Electrophysiology, vol. 6, no. 2, pp. 327-333, 2013.

[24] G. Ciconte, V. Velagić, G. Mugnai et al., "Electrophysiological findings following pulmonary vein isolation using radiofrequency catheter guided by contact-force and second-generation cryoballoon: lessons from repeat ablation procedures," Europace, vol. 18, no. 1, pp. 71-77, 2016. 


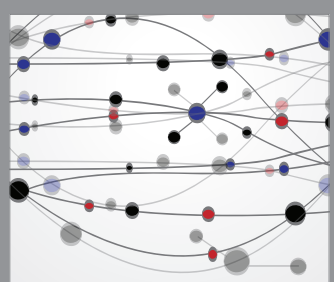

The Scientific World Journal
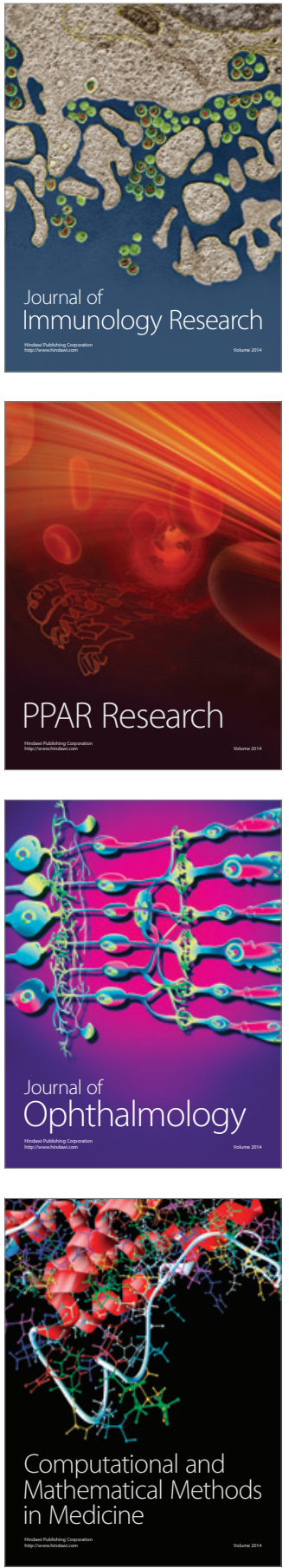

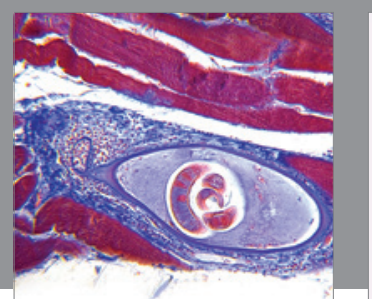

Gastroenterology Research and Practice

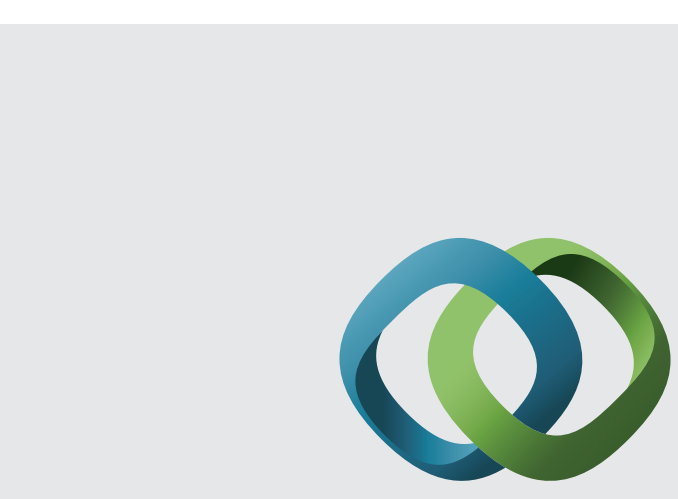

\section{Hindawi}

Submit your manuscripts at

http://www.hindawi.com
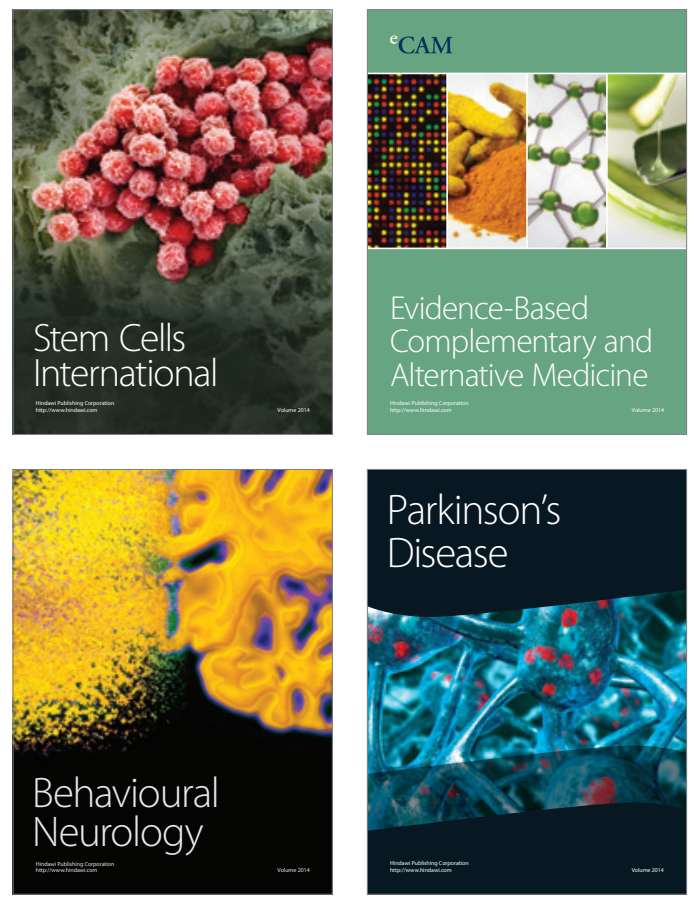
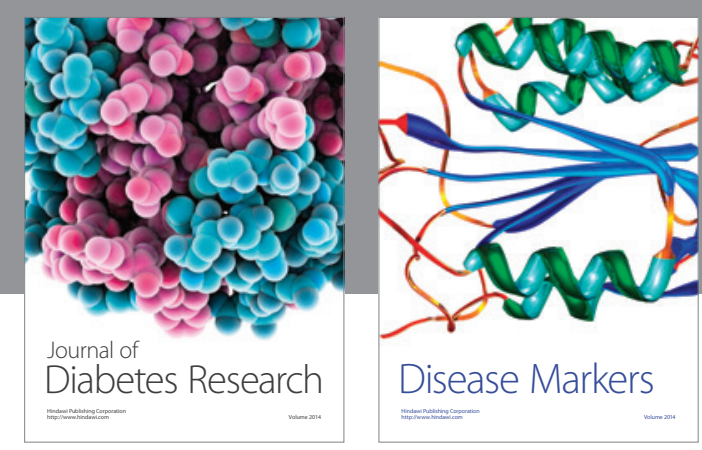

Disease Markers
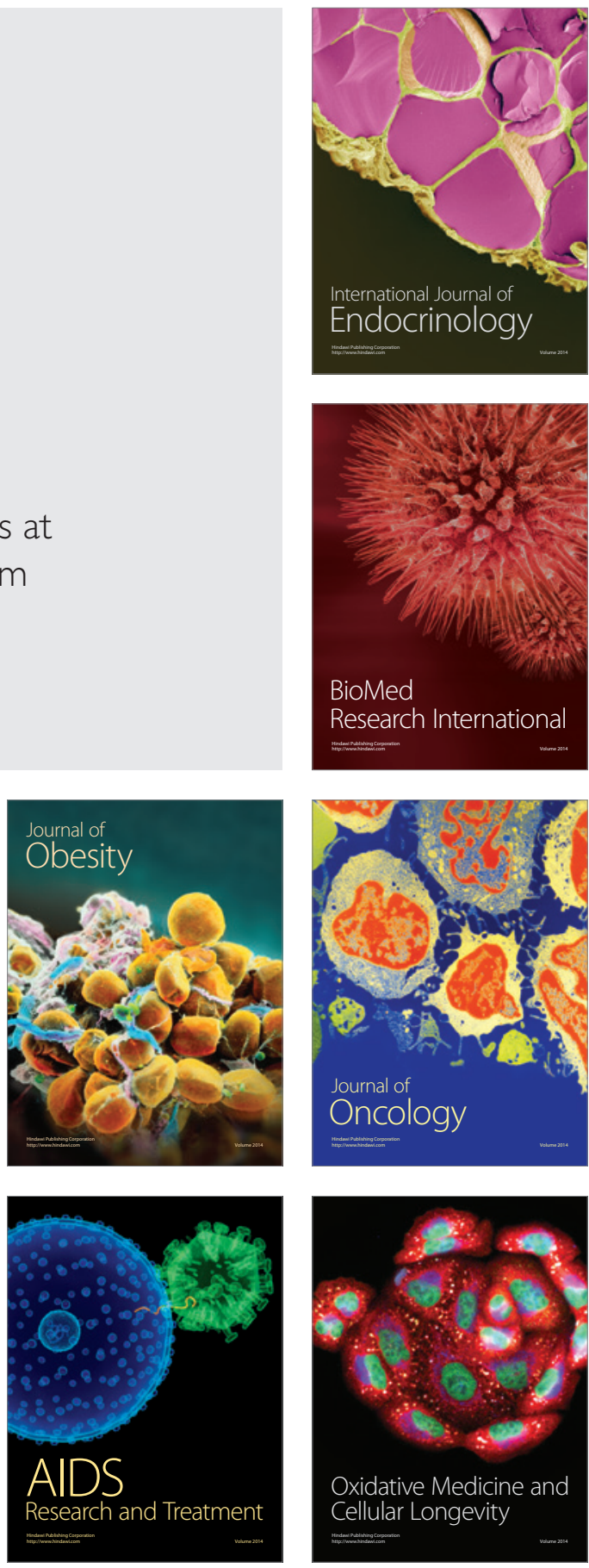